OPEN ACCESS

Edited by:

Gee W. Lau,

University of Illinois at Urbana-

Champaign, United States

Reviewed by:

Luchang Zhu,

Houston Methodist Research Institute,

United States

Qing Deng,

Purdue University, United States

${ }^{*}$ Correspondence:

William F. C. Rigby

william.f.c.rigby@dartmouth.edu

Specialty section:

This article was submitted to Microbial Immunology, a section of the journal

Frontiers in Immunology

Received: 27 January 2021 Accepted: 07 April 2021 Published: 26 April 2021

Citation:

Theprungsirikul J, Skopelja-Gardner S,

Burns AS, Wierzbicki RM and

Rigby WFC (2021) Bactericidal/

Permeability-Increasing Protein

Preeminently Mediates Clearance of

Pseudomonas aeruginosa In Vivo via

CD18-Dependent Phagocytosis.

Front. Immunol. 12:659523. doi: 10.3389/fimmu.2021.659523

\section{Bactericidal/Permeability-Increasing Protein Preeminently Mediates Clearance of Pseudomonas aeruginosa In Vivo via CD18- Dependent Phagocytosis}

\author{
Jomkuan Theprungsirikul ${ }^{1}$, Sladjana Skopelja-Gardner ${ }^{2}$, Ashley S. Burns ${ }^{1}$, \\ Rachel M. Wierzbicki ${ }^{1}$ and William F. C. Rigby ${ }^{1,2 *}$ \\ ${ }^{1}$ Department of Microbiology and Immunology, Geisel School of Medicine at Dartmouth, Lebanon, NH, United States, \\ ${ }^{2}$ Division of Rheumatology, Department of Medicine, Geisel School of Medicine at Dartmouth, Lebanon, NH, United States
}

Chronic Pseudomonas aeruginosa infection mysteriously occurs in the airways of patients with cystic fibrosis (CF), bronchiectasis (BE), and chronic obstructive pulmonary disease (COPD) in the absence of neutrophil dysfunction or neutropenia and is strongly associated with autoimmunity to bactericidal permeability-increasing protein (BPI). Here, we define a critical role for BPI in in vivo immunity against $P$. aeruginosa. Wild type and BPI-deficient (Bpi-/-) mice were infected with $P$. aeruginosa, and bacterial clearance, cell infiltrates, cytokine production, and in vivo phagocytosis were quantified. Bpi-/- mice exhibited a decreased ability to clear $P$. aeruginosa in vivo in concert with increased neutrophil counts and cytokine release. Bpi-/- neutrophils displayed decreased phagocytosis that was corrected by exogenous BPI in vitro. Exogenous BPI also enhanced clearance of $P$. aeruginosa in Bpi-/- mice in vivo by increasing $P$. aeruginosa uptake by neutrophils in a CD18-dependent manner. These data indicate that BPI plays an essential role in innate immunity against $P$. aeruginosa through its opsonic activity and suggest that perturbations in BPI levels or function may contribute to chronic lung infection with $P$. aeruginosa.

Keywords: Bactericidal/permeability-increasing protein (BPI), Pseudomonas, phagocytosis, CD18, neutrophils, inflammation, innate immunity, opsonization

\section{INTRODUCTION}

Polymorphonuclear cells or neutrophils (PMNs) are an essential component of innate immunity against Pseudomonas aeruginosa infection $(1,2)$. Neutropenic mice are more susceptible to $P$. aeruginosa-related mortality (2), while humans deficient in neutrophil-mediated antimicrobial mechanisms (typically altered phagocytic function) have markedly increased susceptibility to $P$. aeruginosa infection $(3,4)$. Thus, immunity against $P$. aeruginosa is dependent on neutrophilmediated phagocytosis. Chronic $P$. aeruginosa infection occurs in the airways of patients with cystic fibrosis (CF), non-CF bronchiectasis (BE), and chronic obstructive pulmonary disease (COPD) in the absence of neutrophil dysfunction or neutropenia (5-10). The underlying mechanism(s) of 
chronic $P$. aeruginosa airway infection in the context of intact neutrophil function is unknown. We previously reported a strong association between autoreactivity to bactericidal/permeabilityincreasing protein (BPI) and chronic $P$. aeruginosa infection in the serum of $\mathrm{CF}$ and $\mathrm{BE}$ patients $(5,6)$. Moreover, levels of autoantibodies to BPI inversely correlate with patient lung function in CF, BE, and COPD (5-9).

In an infected airway, neutrophils mediate killing of $P$. aeruginosa through phagocytosis, neutrophil extracellular trap (NET) formation, and neutrophil degranulation to release bactericidal proteases (11-13). BPI is an antimicrobial agent within neutrophil azurophilic granules that mediates membrane injury of gram-negative bacteria (GNB) following its high affinity binding to lipopolysaccharides (LPS) (14-16). This bactericidal activity of BPI has been recognized in vitro and is transduced by the N-terminal BPI domain (15, 17-22). Mouse BPI shows 53\% amino acid identity and $71 \%$ similarity to human BPI; overexpression of mouse BPI in human embryonic kidney 293 cells resulted in antibacterial activity against Escherichia coli, comparable with that obtained with human BPI (23). The ability of BPI to mediate E. coli opsonization has been reported in vitro and attributed to the ability of the BPI C-terminal domain to engage myeloid cells through an undetermined pathway (17). The function of BPI in innate immunity against GNB in vivo has not been studied.

We demonstrated that BPI is required for clearance of $P$. aeruginosa in vivo, in both lung and peritoneal infection models. The absence of BPI resulted in increased inflammation, characterized by enhanced neutrophil recruitment and inflammatory cytokine production in both the lung and the peritoneum. In the absence of BPI, altered $P$. aeruginosa clearance was due to impaired phagocytosis of $P$. aeruginosa by BPI-deficient neutrophils and not due to an impairment in degranulation or production of reactive oxygen species (ROS). Exogenous human BPI decreased bacterial load and reduced inflammation in vivo by facilitating $P$. aeruginosa uptake into Bpi-/- neutrophils, in a CD18-dependent manner. Together, these findings provide the first in vivo evidence that BPI is essential for eliminating $P$. aeruginosa infection. Moreover, this activity of BPI is transduced through enhanced phagocytosis of $P$. aeruginosa, rather than by direct bacterial cytotoxicity.

\section{METHODS}

\section{Mice}

Age-matched male and female (6-10 weeks old) mouse littermates on the $\mathrm{C} 57 \mathrm{BL} / 6$ background were used for all experiments. Heterozygous Bpi+/- (C57BL/6NJ-Bpi ${ }^{\text {em1(IMPC)J/ }}$ Mmjax) mice were obtained from The Jackson Laboratory (MMRRC Stock: 42125-JAX, Bar Harbor, ME) and bred in our facility. This study was carried out in strict accordance with the recommendations in the Guide for the Care and Use of Laboratory Animals of the National Research Council (24). All experiments were conducted according to protocols approved by Dartmouth College's Institutional Animal Care and Use Committee (IACUC Protocol 00002007). Tail DNA genotyping was performed on pups between 8-14 days of age (Supplemental Figure 1) following the specific strain protocol provided by The Jackson Lab (MMRRC Stock: 42125-JAX). No developmental phenotype was observed with homozygous Bpi-/- mice.

\section{Murine Neutrophil Isolation and Lysate Generation}

For isolation of murine neutrophils, naïve mice (wt or Bpi-/-) were injected intraperitoneally (i.p.) with $1 \mathrm{ml}$ of $4 \%$ thioglycollate solution. Cells were harvested by peritoneal lavage ( $3 \mathrm{ml} \mathrm{PBS)} 12-$ $18 \mathrm{~h}$ later, a time of peak neutrophil induction $(25,26)$. Cells were spun down (1000rpm for 5mins) and erythrocyte lysis performed using 1X BD Pharm Lyse (BD Bioscience). To generate cell lysate, neutrophil pellet was resuspended in citrate-phosphate buffer $\left(0.2 \mathrm{M} \mathrm{Na}_{2} \mathrm{HPO}_{4}+0.1 \mathrm{M}\right.$ citric acid, $\left.\mathrm{pH} 3.0\right)$ and frozen/thawed three times (6). Lysate protein concentration was determined by Pierce BCA Protein Assay Kit (ThermoFisher).

\section{BPI Detection by Immunoblot and Immunofluorescence (IF)}

Immunoblotting against mouse or human BPI $(0.5 \mu \mathrm{g}$ purified $\mathrm{mBPI}$ or $20 \mu \mathrm{g}$ mouse neutrophil protein extract, generated by repeated freeze-thaw cycles in citrate-phosphate buffer) was performed after resolution by SDS-PAGE (12\% acrylamide gel) and transfer to a nitrocellulose membrane. Following blocking in Tris buffered saline with $0.05 \%$ tween-20 (TBST) $+3 \%$ bovine serum albumin (BSA), the membranes were probed with affinity purified mouse BPI antibody (POCONO, amino acid 256-269, $0.1 \mu \mathrm{g} / \mathrm{ml}$ ), anti-hBPI antibody (Santa Cruz, amino acid 227-254, $1: 1000$ ) or mouse beta actin antibody (Cell Signaling, 1:1000) in TBS-T $+1 \%$ BSA overnight at $4^{\circ} \mathrm{C}$, washed, and incubated with goat anti-mouse or anti-rabbit peroxidase-labeled secondary antibody (1:50000 in TBS-T + 1\% BSA). Secondary antibodyonly blots were performed as controls (no signal was detected). SuperSignal West Pico (ThermoFisher) was used for protein detection via the Syngene G-Box system and software (Synoptics).

To detect mouse BPI by IF, thioglycolate-elicited peritoneal neutrophils $(250,000$ cells in $0.5 \mathrm{ml} \mathrm{RPMI}+10 \% \mathrm{FBS})$ that had been collected by lavage were plated onto $13 \mathrm{~mm}$ coverslips for 1 h. Samples were fixed with $4 \%$ paraformaldehyde (PFA), washed, permeabilized using $0.5 \%$ Triton X-100, and blocked using 5\% donkey serum (Sigma-Aldrich). Mouse BPI was detected using rabbit anti-mouse BPI antibody (1:200, ABclonal), followed by donkey anti-rabbit Alexa Fluor 647 or donkey anti-rabbit Alexa Fluor 488 (1:500, Abcam). Samples were mounted using ProLong Gold Antifade Mount with DAPI (ThermoFisher Scientific) and visualized with the laser point scanning confocal microscope (ZEISS LSM 800, Zeiss), 63X. To detect human BPI by IF from in vivo administration of BPI, peritoneal cells were plated onto $13 \mathrm{~mm}$ coverslips, fixed, permeabilized, and blocked as mentioned above. Human BPI was detected using anti-human BPI antibody against aa227-254 (1:200, Santa Cruz), followed by donkey anti-mouse Alexa Fluor 488 (1:500, Jackson ImmunoResearch). 


\section{Bacterial Culture and Phagocytosis Assays}

P. aeruginosa strains PAO1 and PA14 (wt and $\Delta$ popB) were obtained from Dr. Brent Berwin. Bacteria were cultured overnight in lysogeny broth $(\mathrm{LB})$ at $37^{\circ} \mathrm{C}$ and sub-cultured for 3 hrs in $\mathrm{LB}(1: 30)$ at $37^{\circ} \mathrm{C}$. Phagocytosis assay of live $P$. aeruginosa bacteria was performed as previously described $(27,28)$. Neutrophils (250,000 in 250ul RPMI, with or without 30mins $37^{\circ} \mathrm{C}$ pre-incubation with human or mouse BPI) were incubated with $P$. aeruginosa (PAO1 or PA14) for $45 \mathrm{mins}$ at $37^{\circ} \mathrm{C}$ at 10 or 25 multiplicities of infection (MOI) and then treated with $250 \mathrm{ul}$ of gentamicin $(200 \mu \mathrm{g} / \mathrm{ml})$ for $20 \mathrm{mins}$ at $37^{\circ} \mathrm{C}$ to kill extracellular bacteria before being pelleted and washed twice in 500ul RPMI. Neutrophils were resuspended in lysis buffer $(0.1 \%$ Triton X-100 in PBS) and lysed contents (10ul) plated on 1.5\% LB agar plate and incubated overnight at $37^{\circ} \mathrm{C}$. Bacterial colonies (colonyforming unit, $\mathrm{CFU}$ ) were counted on the following day, representing the number of live bacteria phagocytosed by the neutrophils. To confirm phagocytosis, neutrophils were treated with GFP-expressing $P$. aeruginosa (PAO1) as above. After incubation with gentamicin and washing step, 200ul of lysis buffer was added to the tubes, mixed, and transferred to a 96-well Flat Clear Bottom Black Polystyrene Microplates (Fisher Scientific). Fluorescence was measured with BioTek Synergy plate reader $(485 / 528 \mathrm{~nm})$.

\section{BPI-LPS Neutralization Assay}

Thioglycollate-recruited murine neutrophils obtained by peritoneal lavage (wt or Bpi-/-, 200,000 cells/well in 50ul DMEM) were treated with purified human BPI (Athens Research and Technology; 0, 100, 300, or 600nM) 30mins at $37^{\circ} \mathrm{C}$ prior to addition of $E$. coli LPS $(0.1 \mathrm{ng} / \mathrm{ml}$, final volume 200ul). Cells were incubated for $4 \mathrm{hrs}$, spun down, and supernatants collected $\left(-80^{\circ} \mathrm{C}\right)$. TNFa concentration was analyzed by mouse TNFa ELISA MAX Deluxe Set (BioLegend).

\section{Intraperitoneal and Oropharyngeal $P$. aeruginosa In Vivo Challenge and Cytokine Analysis}

For in vivo intraperitoneal (i.p.) and oropharyngeal exposure sublethal infection, mice (wt or Bpi-/-) were anesthetized with isoflurane (to effect) and either injected with $3 \times 10^{6} \mathrm{CFU}$ of subcultured PA14 or PBS i.p. (300ul) or by oropharyngeal administration (50ul). For those receiving BPI treatment, $10 \mu \mathrm{g}$ of human BPI in PBS (Athens Research) was injected i.p. 15mins following bacterial infection. Mice were euthanized $3 \mathrm{hrs}$ postinfection (hpi). Peritoneal and bronchoalveolar lavage (BAL) fluids were collected using $1.5 \mathrm{ml}$ and $0.8 \mathrm{ml}$ PBS, respectively and 10 ul plated (1:10 dilution peritoneal lavage; no dilution $\mathrm{BAL})$ on $1.5 \% \mathrm{LB}$ agar plate. Plates were incubated overnight at $37^{\circ} \mathrm{C}$ and recovered CFUs determined by colony counts that were backcalculated to the original lavage volume. Excess peritoneal and BAL samples were spun down (3000rpm 5 mins) and cell-free lavages stored at $-80^{\circ} \mathrm{C}$ for cytokine analysis. Lysates from cell pellets were made in citrate-phosphate buffer as mentioned above and stored for immunoblotting. Blood samples were collected from the inferior vena cava and serum extracted. TNF $\alpha$, IL-1 $\beta$, and IL- 6 concentrations in the lavage and serum samples were determined with mouse ELISAs: TNFa MAX Deluxe Set (BioLegend), IL-1 $\beta$ Duoset, and IL-6 Duoset (R\&D Systems).

\section{Quantification of Neutrophils and MPO From In Vivo Challenge}

Following in vivo challenge, cells from collected lavages were brought up in PBS to count and stained for flow cytometry analysis. Cells were washed in $1 \mathrm{ml}$ of PBS $+1 \%$ BSA $\left(10^{6}\right.$ cells/ 100ul, treated with 500ul mouse Fc Block (1:200, 10 min, $\left.4^{\circ} \mathrm{C}\right)$, and stained $\left(30 \mathrm{~min}, 4^{\circ} \mathrm{C}\right)$ with mouse specific antibodies (IgG) purchased from Biolegend or Invitrogen (anti-CD45-FITC (1:400), anti-Ly6C-APC (1:200), anti-Ly6G-PE (1:200), antiCD11b-PerCP-Cy5.5 (1:200)). Cells were fixed in 1\% PFA and analyze by flow cytometry (Gallios, with Kaluza Analysis or FlowJo software). Compensation was done using UltraComp eBeads (Invitrogen). Number of neutrophils (CD45+CD11b+ Ly $6 \mathrm{C}^{\text {int }}$ Ly $6 \mathrm{G}^{\text {hi }}$ ) in lavage samples was determined based on total cell count in the samples, normalized to volume collected. Data from BAL was presented as \%Bpi-/-. Mouse Myeloperoxidase DuoSet ELISA (R\&D Systems) was used to measure MPO concentration in lavage samples (1:10).

Murine hind limb long bone dissection and bone marrow isolation was performed as described previously (29). Briefly, mice were euthanized using $\mathrm{CO}_{2}$ according to IACUC protocol and guidelines. Following femoral extraction, bone marrow cells were flushed out with cold PBS and a 22-gauge needle. Cells from the perfused bone marrow were passed through a $70-\mu \mathrm{m}$ filter cell strainer. Erythrocytes were lysed using 1X BD Pharm Lyse (BD Bioscience). Staining and flow cytometry were performed as mentioned above.

\section{In Vivo Phagocytosis Blocking via CD18 Receptor}

Blocking of phagocytosis was achieved by i.p. injection of antiCD18 M18/2 antibody or a rat IgG2b isotype control $(50 \mu \mathrm{g}$, Bio $\mathrm{X}$ Cell) 15 mins after mice were infected with $3 \times 10^{6} \mathrm{CFU}$ of $P$.

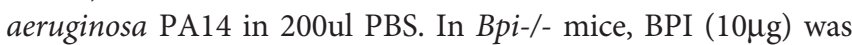
administered i.p. 15 mins following the antibody injection. After 1.5hpi, i.p. lavage was performed (due to unequal immune cellrecruitment at 3 hpi, shorter experiment was performed here). Cell-free lavage was plated onto LB agar plates for $P$. aeruginosa colony count. Peritoneal immune cells were counted to ensure comparable cell recruitment into the peritoneal cavity.

\section{Lung Histology}

Mice (wt and Bpi-/-) infected via oropharyngeal route (noninvasive) with $P$. aeruginosa (PA14, $3 \times 10^{6}$ in 50ul PBS, 3hpi) were sacrificed and lungs harvested and fixed in 10\% formalin (Fisher Scientific) for at least $24 \mathrm{hrs}$. Paraffin sections were generated and stained with Hematoxylin and eosin (H\&E) by the Pathology Research Resource at Dartmouth-Hitchcock Medical Center. The slides were imaged using optical microscope (Olympus BX50), at 40x magnification. 


\section{Quantification of Reactive Oxygen Species (ROS)}

Thioglycollate-induced neutrophils from wt and Bpi-/- mice were stained with DCFDA dye (Cellular ROS Assay Kit, Abcam) for $30 \mathrm{mins}$ at $37^{\circ} \mathrm{C}$ in the dark, washed, and resuspended in $1 \mathrm{X}$ supplemented buffer (supplied with the kit) with 10\% FBS. Stained neutrophils $(100,000$ cells/well) were left untreated or were treated with ROS inducers: tert-butyl hydrogen peroxide (TBHP, 100uM) or glucose oxidase (GO, $2 \mathrm{U} / \mathrm{ml}$ ) for $4 \mathrm{hrs}, 37^{\circ} \mathrm{C}$. Fluorescence was measured at 485/535 nm (BioTek, Synergy HT) $\mathrm{nm}$ in end point mode, with sensitivity 50 and bottom read.

\section{Quantification of Extracellular DNA Release}

Thioglycollate-induced neutrophils from wt and Bpi-/- mice $(200,000$ cells/100 $\mu \mathrm{l})$ were treated with PA14 (MOI 10) or PAO1 (MOI 10) in DMEM without serum (unopsonized) for 3 hrs, $37^{\circ} \mathrm{C}$. Amount of extracellular DNA (ng) was quantified by Sytox Green ( $5 \mu \mathrm{M}$, Invitrogen) and fluorescence measurement (485/530 nm, BioTek, Synergy HT).

\section{Lung Tissue Homogenization}

Mice were infected oropharyngeally with $P$. aeruginosa PA14wt ( $3 \times 10^{6}$ CFU in 50ul PBS) or just PBS for 3hrs. Euthanized mouse lungs were perfused with $5 \mathrm{~mL}$ of ice-cold PBS injected through the right ventricle of the heart. Perfused lungs were removed and placed in RPMI on ice until processing. Lungs were minced and placed in digestion buffer of $1 \mathrm{mg} / \mathrm{mL}$ collagenase type $\mathrm{I}+60 \mathrm{U}$ DNase type I (Sigma-Aldrich) in DPBS Ca2+ Mg2+ (HyClone). Lungs were digested for $30 \mathrm{~min}$ at $37^{\circ} \mathrm{C}$ while shaking, and passed through a $70-\mu \mathrm{m}$ filter cell strainer. Digested contents of the lungs were plated on $\mathrm{LB}$ agar plates to determine recovery CFU. Cells were washed (in RPMI, $1500 \mathrm{rpm}, 4^{\circ} \mathrm{C}$ ) and residual red blood cells were lysed using 1X BD Pharm Lyse lysis buffer (BD Biosciences), for $2 \mathrm{~min}$, and counted. Cells were washed and subsequently blocked using mouse Fc Block (1:200, $\left.10 \mathrm{~min}, 4^{\circ} \mathrm{C}\right)$. Staining for flow cytometry was performed as mentioned above.

\section{Murine BPI (mBPI) Production and Affinity Purification of $\mathrm{mBPI}$ Peptide-Specific Antisera}

Murine BPI plasmid was obtained from Origene (Cat. MR219864). Plasmid was transfected into TOP10 comp cells and DNA was purified using Qiagen Maxi Prep kit (Qiagen) according to manufacturer's instruction. DNA was transfected into Expi293 cells using Expi293 Expression System Kit (Thermo Fisher Cat. A14635) according to manufacturer's instruction. Mouse BPI was purified using Anti-FLAG Affinity Gel (Bimake, Cat. B23101) according to manufacturer's instruction. Protein concentration was measured by the Pierce BCA Protein Assay Kit (ThermoFisher Scientific). Murine BPI was detected by immunoblotting using affinity purified rabbit anti-BPI raised against the murine-specific amino acid (256-269) antibody (Pocono Rabbit Farm \& Laboratory (PRF\&L), Canadensis, PA).

\section{P. aeruginosa Killing Assay by BPI}

BPI $(5 \mu \mathrm{g} / \mathrm{ml}$ or $90 \mathrm{nM})$ was incubated with $10^{8} \mathrm{CFU}$ of PAO1 (in $100 \mathrm{ul} \mathrm{PBS})$. After $30 \mathrm{mins}$ at $37^{\circ} \mathrm{C}$ incubation, the samples were diluted 1:10000 in ice-cold LB before plated (10 $\mu \mathrm{l})$ onto LB agar plates. Plates were incubated overnight at $37^{\circ} \mathrm{C}$ before $\mathrm{CFUs}$ were counted. CFU data was normalized to no-BPI treatment (100\% survival).

\section{Statistical Analyses}

Data were analyzed using GraphPad Prism 6 software (GraphPad Software, Inc., California, USA). Student's paired and unpaired t-tests with Welch's correction were applied to determine the significant difference between two data sets. Oneway ANOVA with multiple comparisons were applied for more than two data sets. $\mathrm{P}<0.05$ was considered significant.

\section{Study Approval}

All experiments were conducted according to protocols approved by Dartmouth College's Institutional Animal Care and Use Committee (IACUC Protocol 00002007).

\section{RESULTS}

\section{BPI Is Required for Efficient $P$. aeruginosa Clearance and Control of Lung Inflammation}

We investigated the role of BPI in immunity against $P$. aeruginosa, using BPI-deficient (Bpi-/-) mice (Supplemental Figure 1). BPI deficiency was confirmed in the Bpi-/- neutrophil lysates, which showed no reactivity when immunoblotted with a mouse-specific BPI (mBPI) antibody, compared to neutrophils from wild type (wt) mice, which demonstrated a $\sim 64 \mathrm{kD}$ band, corresponding to that seen with recombinant $\mathrm{mBPI}$ (Figure 1A and Supplemental Figures 2A, B). Immunofluorescence staining of thioglycollaterecruited peritoneal neutrophils confirmed cytoplasmic residence of BPI in wt and absence of BPI in Bpi-/- neutrophils (Figure 1B).

The role of BPI in the clearance of $P$. aeruginosa in vivo has not been reported. We therefore asked if BPI is required for the clearance of $P$. aeruginosa (PA14) following acute (3hr) infection. Mice, wt and Bpi-/-, were infected with $P$. aeruginosa via intraperitoneal or oropharyngeal routes. Colony forming units (CFU) were assayed in the lavage (peritoneal or bronchoalveolar, respectively) or lung homogenate (Figure 1C). Higher numbers of colony forming units (CFUs) were recovered from both the peritoneal fluid and the bronchoalveolar lavage (BAL) samples of Bpi-/- mice, compared to wt littermates (Figures 1D, E). Bpi-/- mice also demonstrated higher CFUs in the whole lung homogenates following acute lung infection with $P$. aeruginosa (Figure 1F). While no substantive differences in lung pathology were observed between the two genotypes at baseline (Supplemental Figure 3), the inflammatory response to $P$. aeruginosa infection in Bpi-/- mice was associated with increased cellularity and inflammation, compared to wt mice through H\&E staining (Figure 1G). These findings reveal that BPI is required for in vivo clearance of $P$. aeruginosa and suggest that lack of $P$. aeruginosa clearance in the absence of BPI leads to excessive lung tissue inflammation. 
A

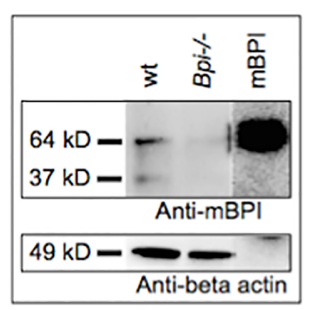

D

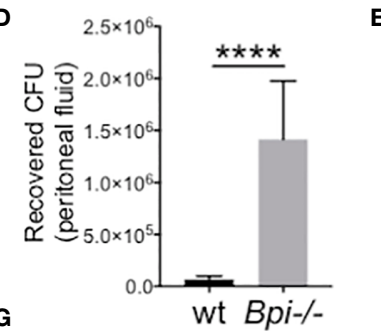

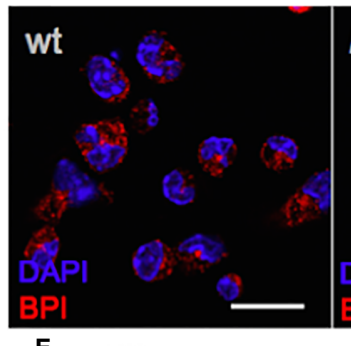

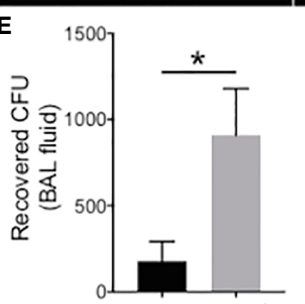

wt Bpi-/-
C

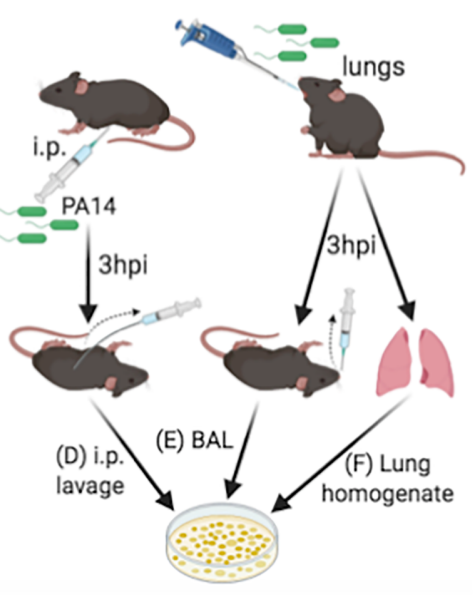

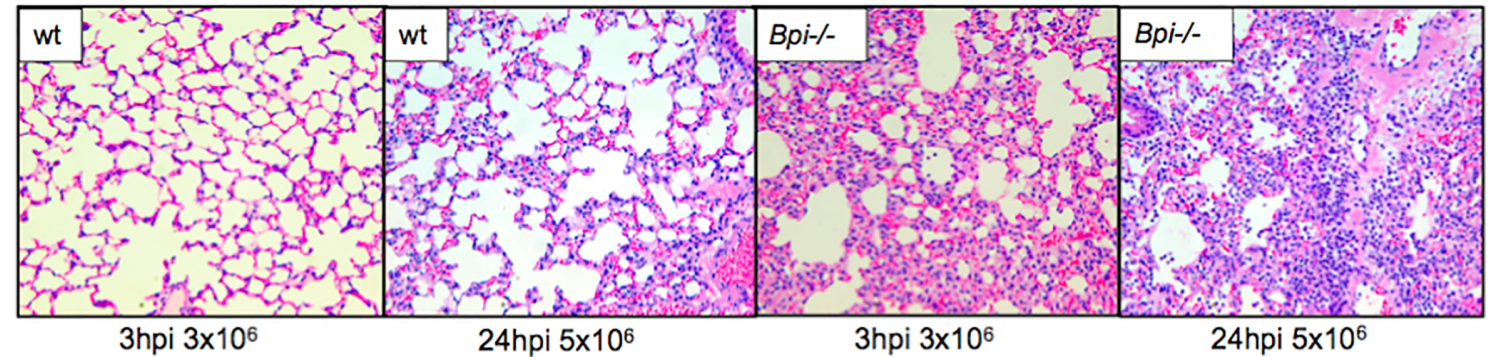

FIGURE 1 | BPI is required for efficient $P$. aeruginosa clearance and control of lung inflammation in vivo. (A) Immunoblots of anti-mouse BPI (mBPI) (amino acids 256-269) and anti-beta actin antibody reactivity to neutrophil lysates (20 $\mu \mathrm{g})$ from wild type (wt) and BPI-deficient (Bpi-/-) mice. Recombinant mBPI (0.5 $\mu \mathrm{g})$ used as control. (B) Immunofluorescence staining of BPI (red) and DAPI (blue) in the peritoneal wt and Bpi-/- mouse PMNs (mPMNs). Images obtained using a 63X oil immersion objective. Scale bar: $20 \mu \mathrm{m}$. (C) Mice were infected with $3 \times 10^{6}$ CFU PA14 via intraperitoneal or oropharyngeal route before lavages or lung homogenate were obtained 3hpi to plate for colony count. (D) Colony forming units (CFU) recovered from peritoneal fluid of mice $(\mathrm{n}=5)$ infected with $P$. aeruginosa PA14 (3x10 ${ }^{6}$

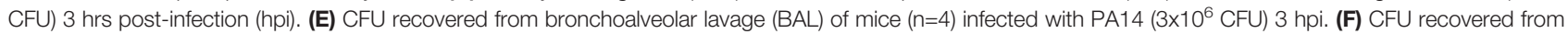

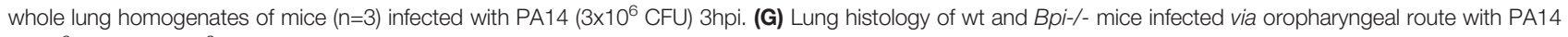
$\left(3 \times 10^{6}\right.$, 3hpi or $\left.5 \times 10^{6}, 24 \mathrm{hpi}\right)$. Representative H\&E staining shown at 40x magnification. Images shown are representative for three samples for each genotype. Statistical significance was determined by Unpaired t-test with Welch's correction, ${ }^{\star \star \star *} \mathrm{p}<0.0001$, ${ }^{\star} \mathrm{p}<0.05$, ns, not significant; Error bars represent mean \pm SEM.

\section{BPI Deficiency Enhances Neutrophil Recruitment and Cytokine Production}

To investigate whether reduced clearance of $P$. aeruginosa in Bpi-/- mice was due to an impaired inflammatory response, we evaluated neutrophil recruitment to the infection site. On the contrary, significantly higher neutrophil numbers were detected in both the BAL and the peritoneum of Bpi-/- mice compared to wt controls following infection (Figures 2A, B). The enhanced neutrophil response in BPI-deficient mice was accompanied by a decrease in the percentage of neutrophils in the bone marrow of Bpi-/- mice (Figure 2C), suggesting greater infection-triggered neutrophil recruitment in the absence of BPI.

The increased neutrophil recruitment in Bpi-/- mice was accompanied by significantly higher concentrations of inflammatory cytokines (TNFa, IL-1b, and IL-6) in both the peritoneal and the BAL fluid samples, compared to the wt mice (up to 3.6 fold increase in peritoneal lavage and 37.4 fold increase in the BAL) (Figures 2D, E). No differences in the peritoneal and the BAL fluid cytokine levels were observed between wt and Bpi-/- mice challenged with PBS, which were overall significantly lower in the absence of PA14 infection (Supplemental Figures 4A, B and Figures 2D, E). Peritoneal infection led to significantly higher levels of serum TNFa, IL-1b, and IL-6 in Bpi-/-, compared to wt mice (Supplemental Figure 5A). With lung infection, a difference in serum cytokines was only seen with IL-1b (Supplemental Figure 5B). Therefore, these data demonstrate that absence of anti-microbial and anti-inflammatory functions mediated by BPI resulted in increased airway, peritoneal and systemic inflammation. Hence, not only is BPI essential for the clearance of $P$. aeruginosa in vivo, intact BPI is required for the control of the inflammatory cellular and cytokine responses during acute $P$. aeruginosa infection.

\section{Compromised $P$. aeruginosa Clearance In Vivo in the Absence of BPI Is Due to Impaired Neutrophil Phagocytosis}

To identify the mechanism(s) responsible for defective bacterial clearance in vivo in the absence of BPI, we investigated different 
A

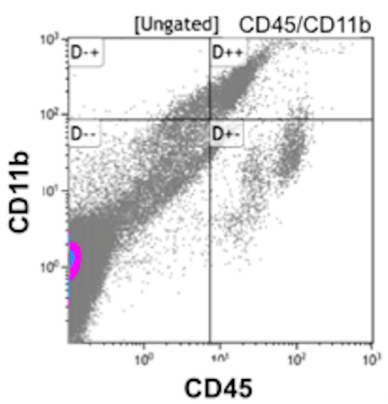

B

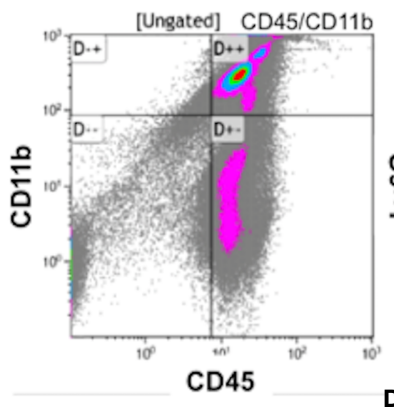

c
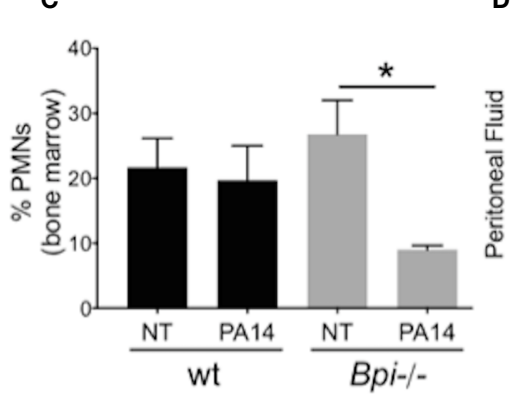
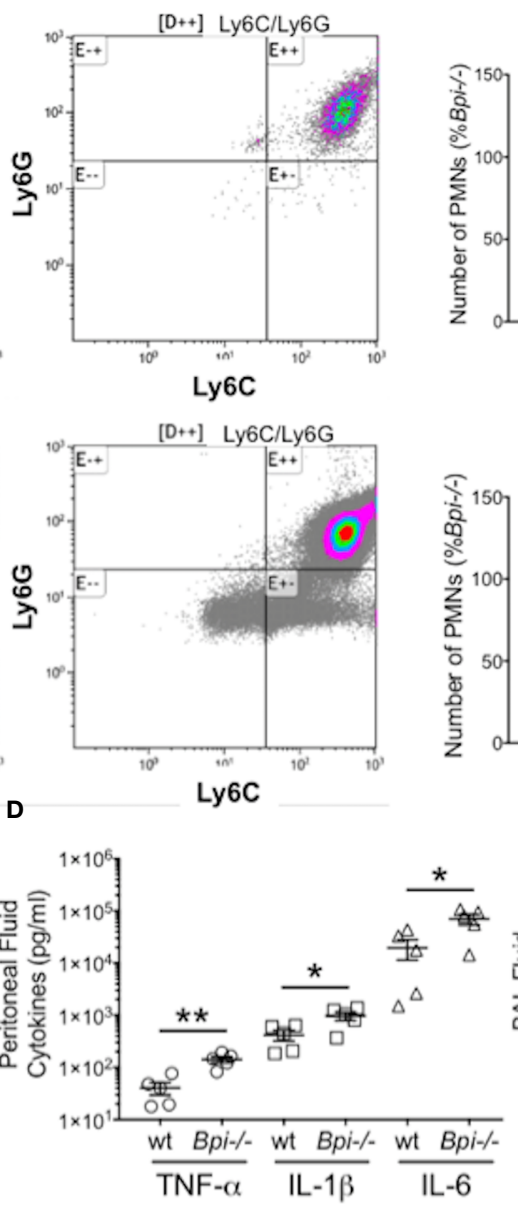

BAL Fluid
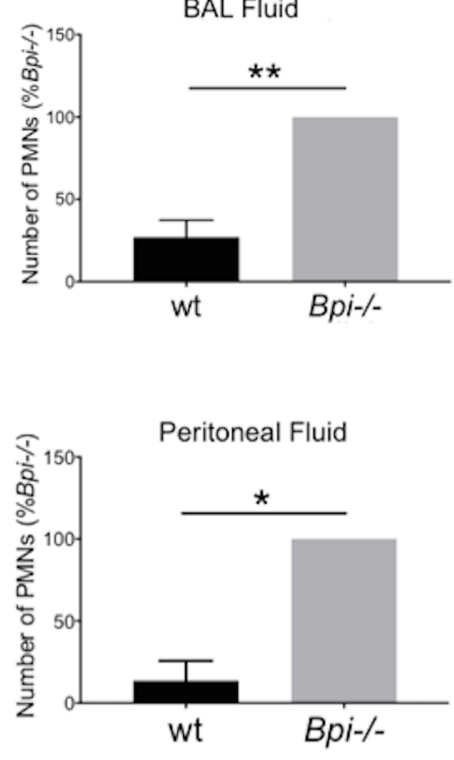

E

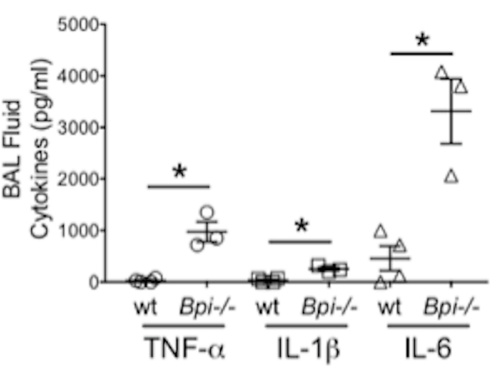

FIGURE 2 | Increased neutrophil recruitment and inflammatory cytokines were observed at infected sites in BPI-deficient mice. (A, B) Neutrophil numbers in the (A) bronchoalveolar lavage and (B) peritoneal lavage of mice infected for 3h with PA14 $\left.3 \times 10^{6} \mathrm{CFU}\right)$ were determined by flow cytometry $\left(\mathrm{CD} 45^{+} \mathrm{CD} 11 \mathrm{~b}^{+} \mathrm{Ly} 6 \mathrm{G}^{+} \mathrm{Ly} 6 \mathrm{C}^{+}\right)$. Bar graphs represent number of neutrophils (PMNs) recovered from wt and Bpi-/- mice $(n=4)$. Cell count was calculated from \% neutrophils from flow cytometry and total number of peritoneal or BAL cells. Representative flow panels from Bpi-/- mice showed the majority of BAL (A, 97.3\%) and peritoneal (B, 90\%) immune cells are neutrophils. (C) Percent bone marrow neutrophils in untreated wt and Bpi-/- mice $(n=4)$ as well as following oropharyngeal infection (PA14, $3 \times 10^{6} \mathrm{CFU}$, 3hpi).

(D, E) Inflammatory cytokines TNF, IL-1b, and IL-6 (pg/ml) were measured in (D) peritoneal fluid ( $\mathrm{n}=5)$ and (E) BAL ( $\mathrm{n}=3,4)$ of mice infected with PA14 (3x10 ${ }^{6}$ CFU, 3hpi). All data were analyzed by unpaired t-test with Welch's correction, except for data in (C) which were analyzed by paired Student $t$-test, ${ }^{* *} p<0.01$, ${ }^{*} p<0.05$; Error bars represent mean \pm SEM.

functions of wt vs. Bpi-/- neutrophils, since the majority of cells recruited to infection site $3 \mathrm{hr}$ after infection are neutrophils (1, 2, Figures 2A, B, e.g. $90 \%$ in the peritoneum and $97.3 \%$ in the BAL). Degranulation was evaluated by quantifying release of myeloperoxidase (MPO), a bactericidal protein residing in neutrophil azurophilic granules alongside BPI (30). MPO levels in the peritoneal lavage fluid were higher in Bpi-/- compared to wt mice (Figure 3A), suggesting that degranulation was not compromised in Bpi-/- neutrophils. Thioglycollate-elicited peritoneal neutrophils were investigated in vitro for differences in reactive oxygen species (ROS) production or phagocytosis ability (Figure 3B). In agreement with their intact ability to undergo degranulation, Bpi-/- neutrophils produced equal levels of neutrophil extracellular traps (NETs) and ROS as the wt neutrophils upon stimulation with $P$. aeruginosa or known ROS stimuli (TBHP-tert-Butyl hydroperoxide or GO-glucose oxidase) (Figure 3C and Supplemental Figure 6).
Together, these data demonstrate that compromised $P$. aeruginosa clearance in BPI-deficient mice in vivo is not due to impaired degranulation or ROS production by Bpi-/- neutrophils.

While cytocidal activity of BPI toward GNB has been reported in vitro $(14-16,31,32)$, its role in neutrophil-mediated phagocytosis of GNB is less well understood $(17,19,20)$. To investigate this, thioglycollate-elicited Bpi-/- and wt neutrophils were assayed for the number of ingested CFUs of $P$. aeruginosa (PAO1 or PA14) in vitro following gentamicin treatment to kill extracellular bacteria. Cell lysates showed significantly fewer $P$. aeruginosa CFUs were recovered from BPI-deficient neutrophils (Figures 3D, E). This effect was independent of the $P$. aeruginosa strain or multiplicity of infection (MOI). To confirm that CFU counts accurately represent relative phagocytic ability of the neutrophils, levels of GFP-expressing $P$. aeruginosa in neutrophil lysates were quantified by fluorescence, following gentamicin 


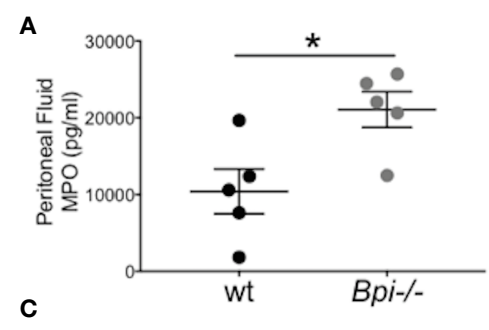

B
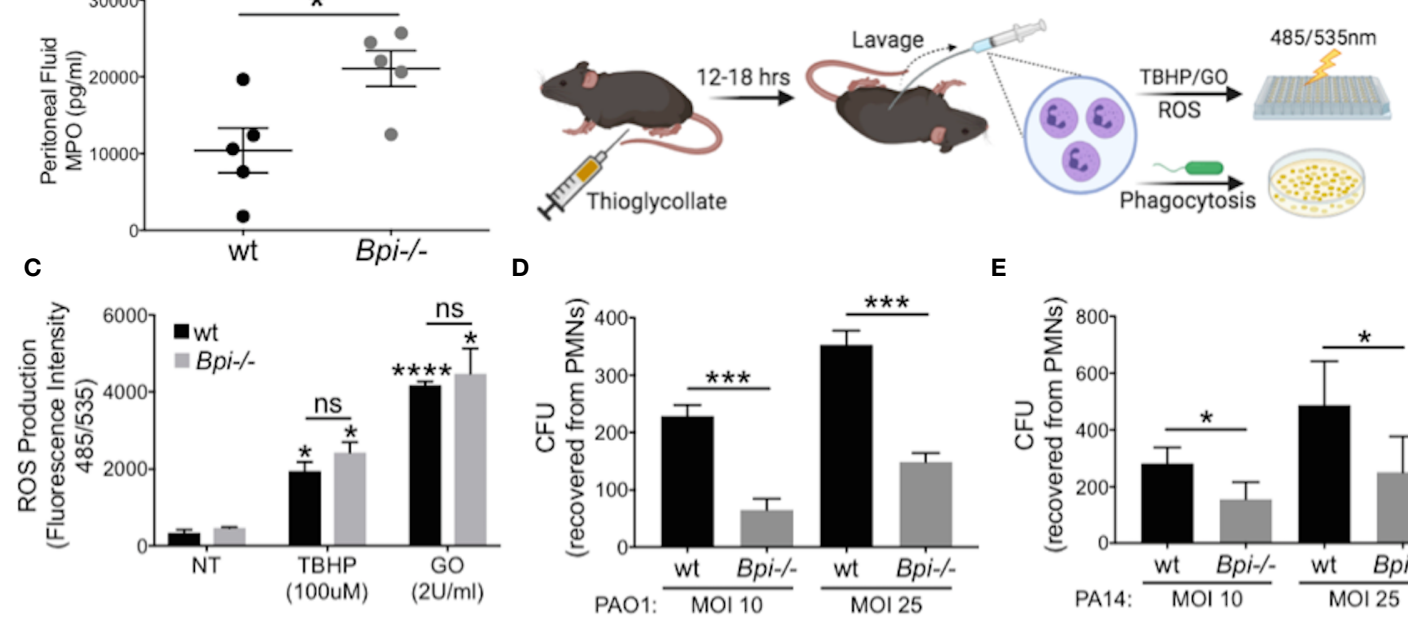

D
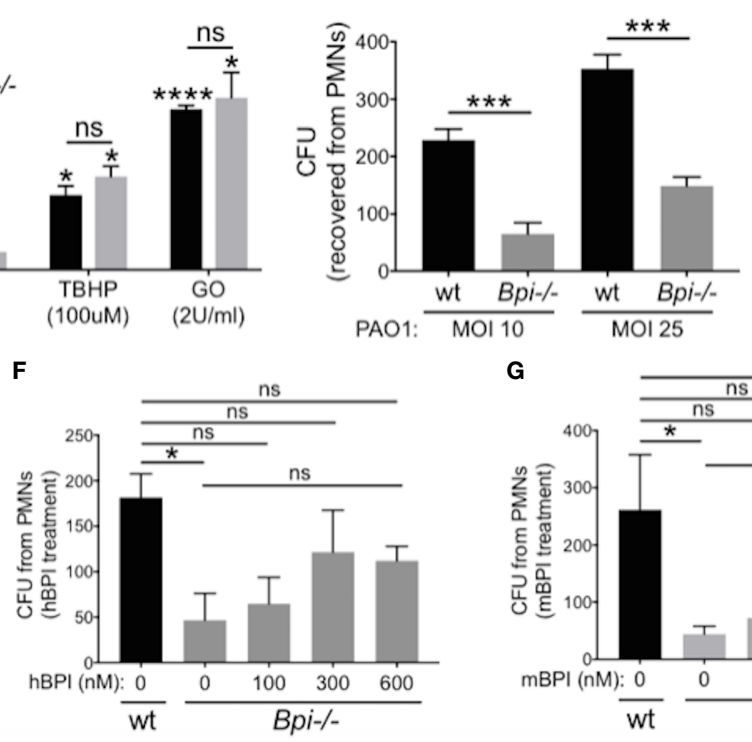

E
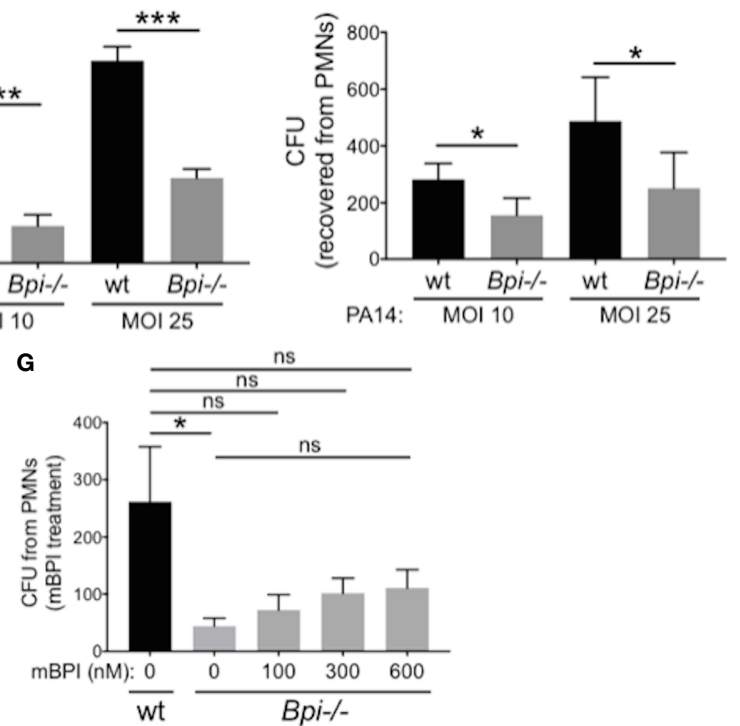

FIGURE 3 | BPI is required for $P$. aeruginosa phagocytosis but not neutrophils degranulation or ROS production. (A) Myeloperoxidase (MPO) concentration, measured by ELISA, in peritoneal fluid of wild type (wt) and Bpi-/- mice ( $n=5)$ infected with PA14 (3hpi). Data were analyzed by unpaired t-test with Welch's correction. (B) Mice were injected intraperitoneally with $1 \mathrm{ml} 4 \%$ thioglycollate, and neutrophils were harvested 12-18 hrs later for reactive oxygen species (ROS) or phagocytosis experiments. (C) ROS production of wt and Bpi-/- PMNs ( $\mathrm{n}=3$ ) after stimulation with tert-Butyl hydroperoxide (TBHP, 100 $\mu$ M) or glucose oxidase (GO, $2 \mathrm{U} / \mathrm{ml}$ ) for $4 \mathrm{hrs}$. Statistical significance was determined relative to untreated (NT) cells ( $\left.{ }^{*} \mathrm{P}<0.05\right)$ or between genotypes (ns, not significant). (D, E) Intracellular colony-forming unit (CFU) counts obtained from lysed wt and Bpi-/- mouse PMNs (mPMNs) challenged with $P$. aeruginosa (D) PAO1 ( $\mathrm{n}=4$ ) and (E) PA14 ( $\mathrm{n}=3$ ) at multiplicity of infection (MOI) of 10 and 25; Statistical significance was determined by paired Student t-test; (F, G) Intracellular CFU counts from wt and Bpi-/mPMNs treated with $P$. aeruginosa PAO1 (MOI 25, $n=3$ ) with increasing concentrations of (F) native human BPI (hBPI, 0-600 nM) or (G) recombinant mouse BPI (mBPI, 0-600 nM). Statistical significance was determined by one-way ANOVA with multiple comparisons for (C), and unpaired t-test with Welch's correction for others. ${ }^{\star \star \star \star} \mathrm{p}<0.0001,{ }^{\star \star *} \mathrm{p}<0.001,{ }^{*} \mathrm{p}<0.05$, ns, not significant; Error bars represent mean \pm SEM.

protection assay. Lower levels of GFP fluorescence intensity was detected in Bpi-/- neutrophil extracts relative to wt controls (Supplemental Figure 7). Addition of exogenous BPI (mouse or human) to Bpi-/- neutrophils prior to in vitro culture with $P$. aeruginosa increased intracellular CFU recovery in a concentration-dependent manner (Figures 3F, G). Moreover, the addition of exogenous BPI neutralized LPS-induced TNFa release by Bpi-/- neutrophils (Supplemental Figure 8). Together, these data suggest that BPI is required for efficient phagocytosis of $P$. aeruginosa by neutrophils in vitro and that it mediates antiinflammatory properties by reducing LPS-induced TNFa release.

\section{Addition of BPI Enhances P. aeruginosa Clearance and Lowers Inflammation in Bpi-/- Mice In Vivo}

To investigate if treatment with BPI can rescue the defect in bacterial clearance seen in Bpi-/- mice in vivo (Figures 1D-F), we administered human BPI (hBPI, 0.2-10 $\mu \mathrm{g}$ ) $15 \mathrm{~min}$ following intraperitoneal $P$. aeruginosa infection of $B p i-/$ - mice (Figure 4A and Supplemental Figure 9). Addition of hBPI significantly reduced $P$. aeruginosa $\mathrm{CFU}$ counts in the peritoneal lavage of Bpi-/- mice, compared to untreated controls (Figure 4B). CFU counts from Bpi-/- mice treated with hBPI were comparable to those of wt mice (Figure 4B). Moreover, treatment of Bpi-/- mice with hBPI resulted in a significant reduction in TNFa, IL- $1 \mathrm{~b}$, and IL-6 levels in the cell-free peritoneal fluid, compared to infected Bpi-/- mice without hBPI treatment (Figure 4C). Full length human BPI $(\sim 64 \mathrm{kD})$ was detected in the lysates of peritoneal immune cells ( $3 \mathrm{~h}$ post infection) by immunoblotting, but not in the cells of untreated Bpi-/- mice (Figure 4D and Supplemental Figure 2C). Immunofluorescence staining confirmed that hBPI was internalized by the infiltrating immune cells, localizing in the cytoplasm (Figure 4E). Exogenous hBPI enabled uptake of $P$. aeruginosa by neutrophils [ $>89 \%$ of CD $45+$ cells in the peritoneum (Figure 2B)] as demonstrated by co-localization of hBPI with td-Tomato expressing $P$. aeruginosa (Figure $4 \mathrm{E}$, top 


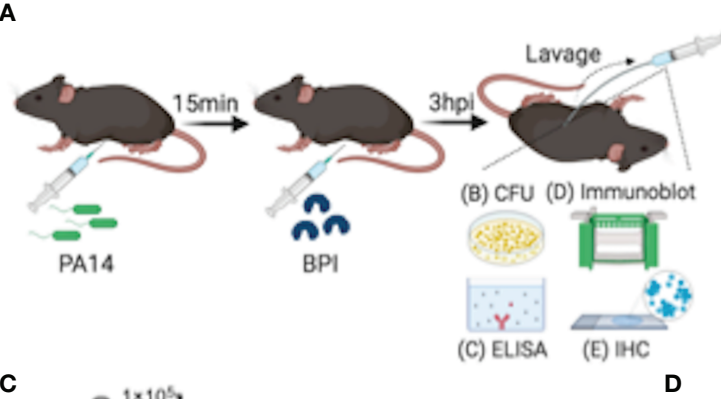

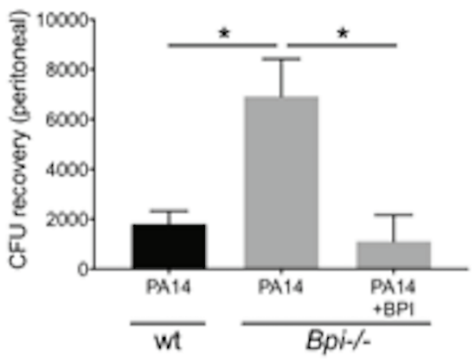

E
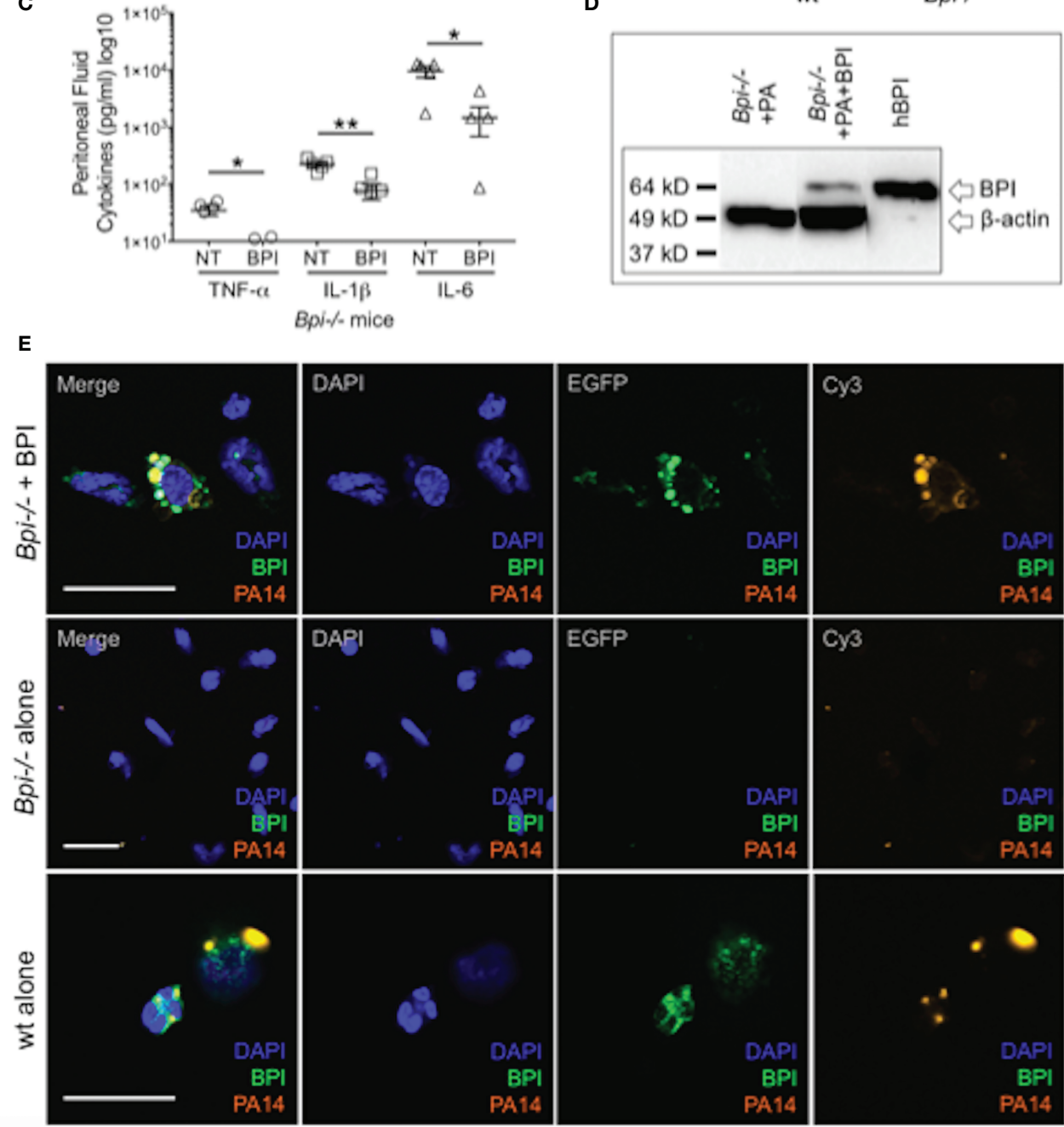

FIGURE 4 | Exogenous BPI mediates P. aeruginosa uptake in Bpi-/- mice in vivo. (A) Mice were administered 10ug BPI 15mins following PA14 infection (3x10 6 $\mathrm{CFU}$ ) via intraperitoneal route. Peritoneal lavage was performed 3hpi for PA14 colony count, ELISA, immunoblot, and IHC. Administration of neutrophil-purified

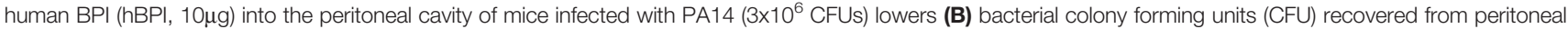
fluid of Bpi-/- mice $(n=4)$, and $(\mathbf{C})$ concentration of pro-inflammatory cytokines found in the peritoneal fluid $(n=5)$. Data were analyzed by unpaired $t$-test with Welch's

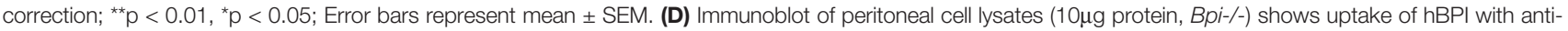
hBPI IgG (amino acids 227-254) following PA14 infection with or without BPI treatment in vivo. Anti-beta actin antibody and recombinant mBPI $(0.05 \mu \mathrm{g})$ were used

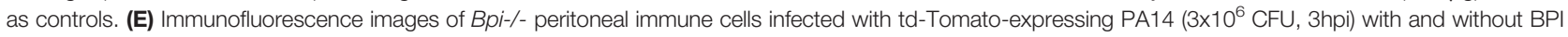
treatment, stained with DAPI (blue) and anti-hBPI antibody (green). Fluorescent images were obtained using a 63X oil immersion objective. Scale bar: $20 \mu \mathrm{m}$. Images shown are representative for three samples for each treatment. 
panels). No td-Tomato expressing $P$. aeruginosa was detected in the peritoneal cells of Bpi-/- mice without hBPI supplementation (Figure 4E, middle panels). Td-Tomato $P$. aeruginosa were observed in the peritoneal cells of wt mice, suggesting that the endogenous BPI also mediates $P$. aeruginosa cellular uptake in vivo (Figure 4E, bottom panels). These findings reveal that administration of exogenous BPI can rescue the ability to clear $P$. aeruginosa in vivo and suggest that a prominent function of BPI in vivo is to mediate phagocytosis of $P$. aeruginosa.

\section{BPI Mediates Clearance of $\boldsymbol{P}$. aeruginosa In Vivo by Phagocytosis, Dependent on CD18}

While prior studies of BPI function, largely limited to in vitro systems, have stressed its direct cytocidal activity $(15,19,20)$, our data suggest an important role for BPI-mediated phagocytosis in the clearance of GNB in vivo (Figure 4). To determine whether the exogenous BPI mediates $P$. aeruginosa clearance in vivo via phagocytosis or direct killing, we blocked the ability of neutrophils to phagocytes $P$. aeruginosa prior to treatment with hBPI by neutralizing CD18, a $\beta 2$ integrin required for the uptake of $P$. aeruginosa by phagocytes (33). Anti-CD18 blocking antibody (clone M18/2) or isotype control was given 15 minutes following
P. aeruginosa (PA14) infection via intraperitoneal route; Bpi-/mice received hBPI $15 \mathrm{mins}$ after antibody treatment, and peritoneal lavage was performed to determine PA14 CFUs (Figure 5A). Anti-CD18 treatment completely abolished the enhanced PA14 clearance mediated by administration of exogenous hBPI (Figure 5B). We validated the ability of antiCD18 antibody to effectively block phagocytosis by treating PA14 infected wt mice and demonstrating higher CFU counts in the lavage, compared to isotype treated controls (Figure 5B). Peritoneal immune cell count in both Bpi-/- and wt mice was comparable between all treatments (Figure 5C), suggesting that the effect seen on peritoneal CFUs (Figure 5B) was not due to disparities in immune cell recruitment. Pseudomonas strains with T3SS can manipulate host cells leading to evasion of phagocytosis and clearance (34). Infecting mice with $P$. aeruginosa strain deficient in Type III secretion system (T3SS) translocon (PA14 1 popB) showed comparable recovered CFU to mice infected with $P$. aeruginosa strain sufficient in T3SS translocon (PA14wt) (Supplemental Figure 11). These two strains of $P$. aeruginosa are equally susceptible to BPI killing in vitro (Supplemental Figure 12). Thus, absence or presence of a functional T3SS exhibited no significant effect on Pseudomonas $\mathrm{CFU}$ in wt and Bpi-/- mice. Together, these data suggest that BPI

A

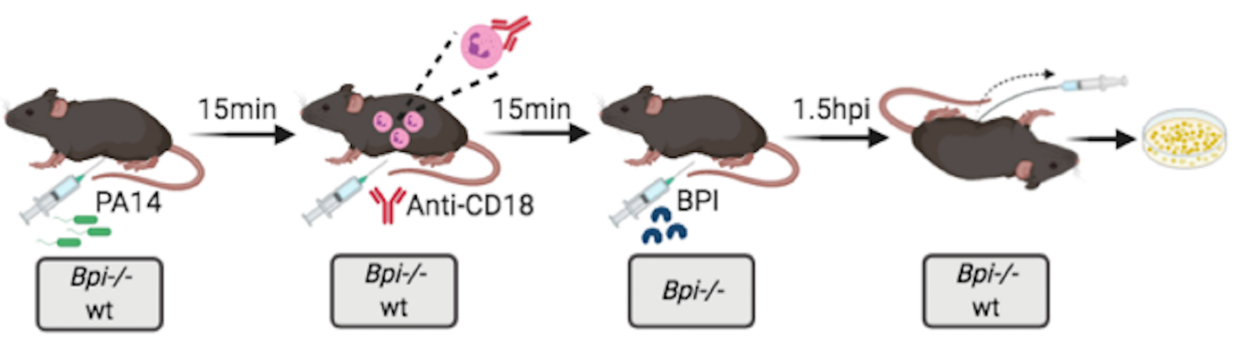

B

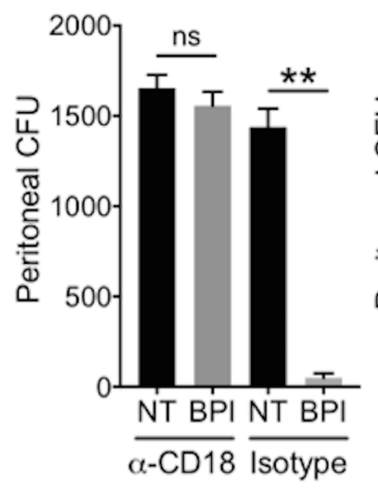

Bpi-/- mice

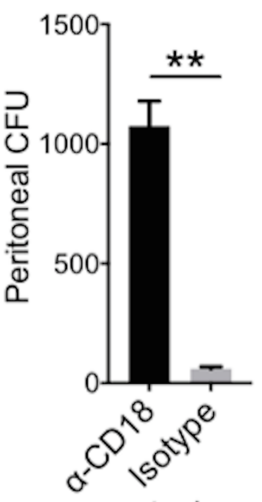

wt mice

C

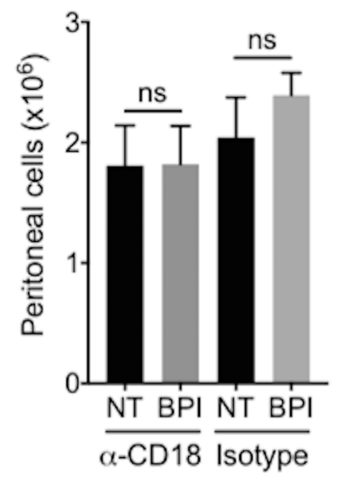

Bpi-/- mice

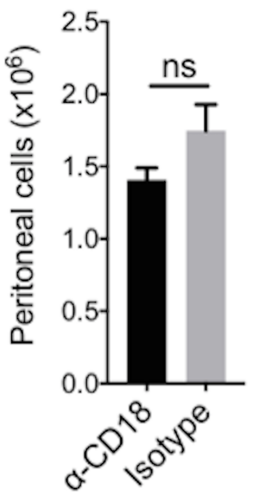

wt mice

FIGURE 5 | BPI mediates $P$. aeruginosa clearance in vivo preeminently by phagocytosis, dependent on CD18. (A) Mice (wt or Bpi-/-) were infected with $3 \times 10^{6}$ CFU

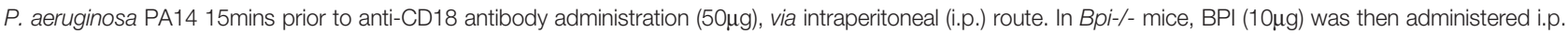
15mins following the antibody treatment. Peritoneal lavage was performed 1.5hrs post-anti-CD18 lgG (wt mice) or post-BPI (Bpi-/- mice) injection, and plated on LB agar for colony counts. (B) Bacterial colony forming units (CFU) recovered from peritoneal fluid after $P$. aeruginosa infection of Bpi-/- mice (left panel) treated with a$\mathrm{CD} 18 \mathrm{lgG}$ or isotype lgG in the absence (NT) or presence of exogenous BPI ( $\mathrm{n}=3$ per treatment group), or wt mice (right panel) treated with a-CD18 IgG or isotype $\lg G(n=3)$. (C) Peritoneal immune cell count in anti-CD18 IgG or isotype treated mice with $P$. aeruginosa infection, with or without BPI administration in Bpi-/- mice $(n=3)$. Data were analyzed by paired t-test with parametric test; ${ }^{* *} \mathrm{p}<0.01$, ns, not significant; Error bars represent mean $\pm \mathrm{SEM}$. 
mediates $P$. aeruginosa clearance in vivo mainly via bacterial phagocytosis and that this process is dependent on CD18.

\section{DISCUSSION}

The selective ability of $P$. aeruginosa to chronically infect the airways in patients with $\mathrm{CF}, \mathrm{BE}$ and COPD in the absence of neutropenia is poorly understood $(5-10)$. We report that BPIdeficient mice exhibit decreased ability to limit $P$. aeruginosa infection in both the lung and peritoneum in vivo. Thus, in this model system, BPI plays a functionally non-redundant role in innate immunity against $P$. aeruginosa. This defect is characterized by increased neutrophil accumulation and production of inflammatory cytokines at sites of infection, propagated by increased recruitment of neutrophils from the bone marrow in Bpi-/- mice. The absence of BPI did not impair neutrophil degranulation or ROS production. Rather, in vitro and in vivo studies demonstrated that Bpi-/- neutrophils exhibited markedly decreased phagocytosis of $P$. aeruginosa. This defect was corrected in vivo by administering exogenous human BPI. Following phagocytosis, ingested $P$. aeruginosa in neutrophils co-localized with exogenous BPI, suggesting a specific and direct role of intact BPI in bacterial opsonization. Lastly, the inability of exogenous BPI to clear $P$. aeruginosa in the presence of CD18 blockade supports the pre-eminent role of opsonization by BPI as well as identifies its transduction via a CD18-dependent pathway.

The anti-bacterial functions of BPI have mainly been studied in vitro in systems without cell interactions (14-17, 19, 20, 31, 32). We confirmed that BPI exhibits direct cytocidal activity towards $P$. aeruginosa in vitro (Supplemental Figure 10). These studies do not recapitulate the complexity and heterogeneity of the in vivo immune cell environment. Our study of BPI-deficient mice is novel, as it is the first study to demonstrate the function of BPI in $P$. aeruginosa phagocytosis and clearance in vivo. Prior in vitro studies have shown that BPI cytotoxic activities reside in the $\mathrm{N}$ terminal domain which also confers LPS binding $(19,20,31,32)$. In contrast, the C-terminal domain is thought to confer opsonic activity of Escherichia coli through an as-yet-undefined pathway in neutrophils and monocytes in in vitro systems $(17,35)$. For the first time, we provide in vivo evidence of BPI-mediated phagocytosis, by demonstrating $P$. aeruginosa co-localization with intact human BPI (confirmed by immunofluorescence) in Bpi-/neutrophils in peritoneal infection. Immunoblotting of neutrophil lysates confirmed the absence of BPI proteolysis with neutrophil uptake, suggesting the requirement for intact BPI. Thus, our data strongly support the model by which the cationic N-terminal portion of intact BPI binds LPS on $P$. aeruginosa while the Cterminal domain mediates neutrophil phagocytosis of an intact BPI-P. aeruginosa complex. Moreover, CD18 blockade inhibits this activity of exogenous human BPI in vivo, thereby confirming that the functional importance of BPI in this model is largely opsonic in nature, rather than by transducing bacterial cytotoxicity.

The ability of CD18 neutralizing antibodies to inhibit BPIdependent phagocytosis in Bpi-/- neutrophils suggests a direct interaction. Recent study has shown that CD18 expression facilitates the uptake of both motile and nonmotile $P$. aeruginosa strains (33). This central role in $P$. aeruginosa uptake has been confirmed; CD18 is the only phagocytic cell surface receptor with genetic evidence to support that it is critical for uptake of $P$. aeruginosa $(36,37)$. CD18 ( $\beta 2$ integrin) forms heterodimers with CD11a, CD11b, CD11c and CD11d (38). Thus, two models are possible: i) BPI alters $P$. aeruginosa membrane structure to allow for CD18 binding, or ii) BPI directly engages with CD18 for binding and phagocytosis of $P$. aeruginosa. Therefore, our findings of the key importance of BPI in mediating $P$. aeruginosa phagocytosis provide a strong rationale to investigate the CD18-BPI interactions in chronic infectious diseases such as $\mathrm{CF}$, non-CF BE, and COPD, where reduced phagocytosis has been reported (39-43). In CF and non-CF BE, decreased phagocytosis by both neutrophils and macrophages has been identified and correlated with reduced expression of phagocytic receptors (39-41). Whether and how this might influence BPI mediated-phagocytosis warrants investigation. In COPD patients, who frequently suffer from airway $P$. aeruginosa infection $(10,44)$, macrophages demonstrate significantly reduced phagocytic capacity of bacteria and apoptotic cells $(42,43,45)$. Since BPI is also expressed by human macrophages albeit at lower levels than neutrophils $(14,46)$, BPI is likely to play an important role in macrophage-mediated phagocytosis. Together, our data suggest BPI as a potential therapy to enhance immune cell-mediated phagocytosis in diseases such as $\mathrm{CF}, \mathrm{BE}$, and COPD.

The observation of a BPI-specific defect in clearing $P$. aeruginosa is notable given its ubiquitous and chronic nature in $\mathrm{CF}, \mathrm{BE}$, and COPD. $P$. aeruginosa infection in these diseases is strongly linked with autoantibodies to BPI (5-10, 47). Moreover, autoantibodies to BPI correlate with worsening lung function in patients with these diseases (5-7). While autoantibodies to BPI are found in other disease states, high-avidity autoantibodies are restricted to patients with chronic lung infection with $P$. aeruginosa $(6,48)$. Given our data that BPI is required for $P$. aeruginosa immunity in vivo, the serologic findings in $\mathrm{CF}, \mathrm{BE}$, or COPD suggest that autoantibodies against BPI might shape a protective niche that enables chronic infection by $P$. aeruginosa in the clinical setting. The unique properties of this model not only derive from the specific association of lung infection by $P$. aeruginosa with BPI autoimmunity but also our demonstration of the importance of BPI in clearing this organism in vivo. Interestingly, no relationship was seen between circulating BPI levels and antibodies to $P$. aeruginosa in a cohort of bacteremic patients (48). This evidence suggests that patients who are susceptible to $P$. aeruginosa infections are not defective in producing or releasing BPI to combat infections. This further supports the notion that the presence of autoantibodies to BPI restricts its function during infection. In addition to bacterial clearance, the therapeutic potential of BPI is also to modulate inflammation, as phagocytosis in its nature is an antiinflammatory process (49). Excessive inflammation leads to lung injury in CF (50) particularly by neutrophils. Our data strongly implicate BPI to be a potent anti-inflammatory molecule, as seen with the levels of cytokines in vivo and lung pathology. This is particularly important in the light of possible diminished BPI 
function in patients with anti-BPI autoantibodies. Studies which confirm a functional role of BPI autoantibodies as well as address their specific association with $P$. aeruginosa lung infection would potentially lead to novel immunomodulatory strategies.

\section{DATA AVAILABILITY STATEMENT}

The raw data supporting the conclusions of this article will be made available by the authors, without undue reservation.

\section{ETHICS STATEMENT}

The animal study protocols were reviewed, approved, and conducted according to Dartmouth College's Institutional Animal Care and Use Committee (IACUC Protocol 00002007).

\section{AUTHOR CONTRIBUTIONS}

JT, WR, and SS-G conceived the project and designed the experiments. JT and RW performed the experiments. AB produced and purified essential reagents. JT and SS-G performed data analyses and interpretation. JT, WR, and SS-G wrote the manuscript. JT prepared the figures. WR supervised all aspects of the study. All authors contributed to the article and approved the submitted version.

\section{REFERENCES}

1. Mizgerd JP. Molecular Mechanisms of Neutrophil Recruitment Elicited by Bacteria in the Lungs. Semin Immunol (2002) Apr14(2):123-32. doi: 10.1006/ smim.2001.0349

2. Koh AY, Priebe GP, Ray C, Van Rooijen N, Pier GB. Inescapable Need for Neutrophils as Mediators of Cellular Innate Immunity to Acute Pseudomonas Aeruginosa Pneumonia. Infect Immun (2009) 77(12):5300-10. doi: 10.1128/ IAI.00501-09

3. Rada B. Interactions Between Neutrophils and Pseudomonas aeruginosa in Cystic Fibrosis. Pathogens (2017) 6(1):10. doi: 10.3390/pathogens6010010

4. Andrews T, Sullivan KE. Infections in Patients With Inherited Defects in Phagocytic Function. Clin Microbiol Rev (2003) 16:597-621. doi: 10.1128/ CMR.16.4.597-621.2003

5. Skopelja-Gardner S, Theprungsirikul J, Meagher RE, Beliveau CM, Bradley $\mathrm{KE}$, Avery M, et al. Autoimmunity to bactericidal/permeability-increasing protein in bronchiectasis exhibits a requirement for Pseudomonas aeruginosa IgG response. Eur Resp Jour (2019) 53(2):1801891. doi: 10.1183/ 13993003.01891-2018

6. Theprungsirikul J, Skopelja-Gardner S, Meagher RM, Clancy JP, Zemanick ET, Ashare A, et al. Dissociation of Systemic and Mucosal Autoimmunity in Cystic Fibrosis. J Cyst Fibros (2020) 19(2):196-202. doi: 10.1016/ j.jcf.2019.06.006

7. Skopelja S, Hamilton BJ, Jones JD, Yang ML, Mamula M, Ashare A, et al. The Role for Neutrophil Extracellular Traps in Cystic Fibrosis Autoimmunity. JCI Insight (2016) 1(17):e88912. doi: 10.1172/jci.insight.88912

8. Hilliam Y, Moore MP, Lamont IL, Bilton D, Charles S H, Foweraker J, et al. Pseudomonas Aeruginosa Adaptation and Diversification in the non-Cystic Fibrosis Bronchiectasis Lung. Eur Respir J (2017) 49(4):1602108. doi: 10.1183/ 13993003.02108-2016

\section{FUNDING}

This work was supported by the Cystic Fibrosis Foundation (CFF) [grant number: RIGBY 20G0], and the BioMT Institute for Biomolecular Targeting core lab (grant: P20GM113132). Further support came from the Translational Research Core at the Dartmouth Geisel School of Medicine, the Shared Resource facility, Irradiation, the Pre-clinical Imaging and Microscopy Resource (IPIMSR) at the Norris Cotton Cancer Center (NCI Cancer Center Support Grant 5P30CA023108-37 and NIH shared instrument grant S10OD21616, for the use of microscopy, pathology and flow cytometry instruments.

\section{ACKNOWLEDGMENTS}

We thank Andreia Isabel Ferreira Verissimo, Sally Demirdjian, Kevin Hsu, Tiffany Coupet, Claire Godbersen-Palmer and Dr. Jack Hoopes for technical assistance, Dr. Brent Berwin for providing the $P$. aeruginosa bacterial strains, and Dr. Robert Homer for lung pathology consultation. All diagrams created with (BioRender.com).

\section{SUPPLEMENTARY MATERIAL}

The Supplementary Material for this article can be found online at: https://www.frontiersin.org/articles/10.3389/fimmu.2021. 659523/full\#supplementary-material

9. Folkesson A, Jelsbak L, Yang L, Johansen HK, Ciofu O, Hoiby N, et al. Adaptation of Pseudomonas aeruginosa to the cystic fibrosis airway: an evolutionary perspective. Nat Rev Microbiol (2012) 10(12):841-51. doi: $10.1038 /$ nrmicro2907

10. Murphy TF, Brauer AL, Eschberger K, Lobbins P, Grove L, Cai X, et al. Pseudomonas aeruginosa in chronic obstructive pulmonary disease. Am J Respir Crit Care Med (2008) 177(8):853-60. doi: 10.1164/rccm.200709-1413OC

11. Pollard AJ, Heale JP, Tsang A, Massing B, Speert DP. Nonopsonic phagocytosis of Pseudomonas aeruginosa: insights from an infant with leukocyte adhesion deficiency. Pediatr Infect Dis J (2001) 20:452-4. doi: 10.1097/00006454-200104000-00019

12. Brinkmann V, Reichard U, Goosmann C, Fauler B, Uhlemann Y, Weiss DS, et al. Neutrophil Extracellular Traps Kill Bacteria. Science (2004) 303:1532-5 doi: 10.1126/science.1092385

13. Timár CI, Lorincz AM, Csépányi-Kömi R, Vályi-Nagy A, Nagy G, Buzás EI, et al. Antibacterial Effect of Microvesicles Released From Human Neutrophilic Granulocytes. Blood (2013) 121:510-8. doi: 10.1182/blood-2012-05-431114

14. Weiss J, Elsbach P, Olsson I, Odeberg H. Purification and Characterization of a Potent Bactericidal and Membrane Active Protein From the Granules of Human Polymorphonu-Clear Leukocytes. J Biol Chem (1978) 253:2664-72. doi: 10.1016/S0021-9258(17)40872-6

15. Gazzano-Santoro H, Parent JB, Grinna L, Horwitz A, Parsons T, Theofan G, et al. High-Affinity Binding of the Bactericidal/Permeability-Increasing Protein and a Recombinant Amino-Terminal Fragment to the Lipid A Region of Lipopolysaccharide. Infect Immun (1992) 60(11):4754-61. doi: 10.1128/IAI.60.11.4754-4761.1992

16. Wang X, Quinn PJ. Endotoxins: Lipopolysaccharides of Gram-Negative Bacteria. Subcell Biochem (2010) 53:3-25. doi: 10.1007/978-90-481-9078-2_1

17. Iovine NM, Elsbach P, Weiss J. An Opsonic Function of the Neutrophil Bactericidal/Permeability-Increasing Protein Depends on Both its $\mathrm{N}$ - and 
C-terminal Domains. Proc Natl Acad Sci U S A (1997) 94(20):10973-8. doi: 10.1073/pnas.94.20.10973

18. Capodici C, Weiss J. Both N- And C-terminal Regions of the Bioactive Nterminal Fragment of the Neutrophil Granule Bactericidal/PermeabilityIncreasing Protein Are Required for Stability and Function. J Immunol (1996) 156(12):4789-96.

19. Ooi CE, Weiss J, Elsbach P, Frangione B, Mannion B. A 25-kDa NH2-terminal fragment carries all the antibacterial activities of the human neutrophil 60$\mathrm{kDa}$ bactericidal/permeability-increasing protein. J Biol Chem (1987) 262:14891-4. doi: 10.1016/S0021-9258(18)48110-0

20. Ooi CE, Weiss J, Doerfler ME, Elsbach P. Endotoxin-Neutralizing Properties of the $25 \mathrm{Kd} \mathrm{N}$-terminal Fragment and a Newly Isolated $30 \mathrm{Kd}$ C-terminal Fragment of the 55-60 Kd Bactericidal/Permeability-Increasing Protein of Human Neutrophils. J Exp Med (1991) 174:649-55. doi: 10.1084/jem.174.3.649

21. Iovine N, Eastvold J, Elsbach P, Weiss JP, Gioannini TL. The CarboxylTerminal Domain of Closely Related Endotoxin-Binding Proteins Determines the Target of Protein-Lipopolysaccharide Complexes. J Biol Chem (2002) 277:7970-8. doi: 10.1074/jbc.M109622200

22. Hume JRDepartment of Microbiology. BPI-mediated delivery of endotoxinrich particles to human $\mathrm{CD14+}$ monocytes: molecular determinants and fate of endotoxin. Iowa City: The University of Iowa (2005). p. 165.

23. Lennartsson A, Pieters K, Vidovic K, Gullberg U. A Murine Antibacterial Ortholog to Human Bactericidal/Permeability-Increasing Protein (BPI) is Expressed in Testis, Epididymis, and Bone Marrow. J Leukoc Biol (2005) Mar77(3):369-77. doi: 10.1189/jlb.0304159

24. National Research Council. Guide for the Care and Use of Laboratory Animals. Washington, DC: National Academies Press (1996).

25. Patankar YR, Mabaera R, Berwin B. Differential ASC Requirements Reveal a Key Role for Neutrophils and a Noncanonical IL-1 $\beta$ Response to Pseudomonas aeruginosa. Am J Physiol Lung Cell Mol Physiol (2015) 309(8): L902-13. doi: 10.1152/ajplung.00228.2015

26. Hermida DRM, Malta R, de S, Santos MDPC, dos-Santos WLC. Selecting the Right Gate to Identify Relevant Cells for Your Assay: A Study of Thioglycollate-Elicited Peritoneal Exudate Cells in Mice. BMC Res Notes (2017) 10:695. doi: 10.1186/s13104-017-3019-5

27. Amiel E, Alonso A, Uematsu S, Akira S, Poynter ME, Berwin B. Pivotal Advance: Toll-Like Receptor Regulation of Scavenger receptor-A-mediated Phagocytosis. J Leukoc Biol (2009) 85:595-605. doi: 10.1189/jlb.1008631

28. Amiel E, Lovewell RR, O'Toole GA, Hogan DA, Berwin B. Pseudomonas aeruginosa evasion of phagocytosis is mediated by loss of swimming motility and is independent of flagellum expression. Infect Immun (2010) 78:2937-45. doi: 10.1128/IAI.00144-10

29. Amend SR, Valkenburg KC, Pienta KJ. Murine Hind Limb Long Bone Dissection and Bone Marrow Isolation. J Vis Exp (2016) 110:e53936. doi: $10.3791 / 53936$

30. Klebanoff SJ. Myeloperoxidase: Friend and Foe. J Leukoc Biol (2005) 77:598625. doi: 10.1189/jlb.1204697

31. Schultz H, Schinke S, Mosler K, Herlyn K, Schuster A, Gross WL. BPI-ANCA of pediatric cystic fibrosis patients can impair BPI-mediated killing of $E$. coli DH5alpha in vitro. Pediatr Pulmonol (2004) 37(2):158-64. doi: 10.1002/ ppul.10416

32. Weinrauch Y, Foreman A, Shu C, Zarember K, Levy O, Elsbach P, et al. Extracellular Accumulation of Potently Microbicidal Bactericidal/ Permeability-Increasing Protein and p15s in an Evolving Sterile Rabbit Peritoneal Inflammatory Exudate. J Clin Invest (1995) 95(4):1916-24. doi: 10.1172/JCI117873

33. Demirdjian S, Hopkins D, Cumbal N, Lefort CT, Berwin B. Distinct Contributions of CD18 Integrins for Binding and Phagocytic Internalization of Pseudomonas aeruginosa. Infect Immun (2020) 88(5): e00011-20. doi: 10.1128/IAI.00011-20

34. Coburn B, Sekirov I, Finlay BB. Type III Secretion Systems and Disease. Clin Microbiol Rev (2007) 20(4):535-49. doi: 10.1128/CMR.00013-07

35. Nishimura H, Gogami A, Miyagawa Y, Nanbo A, Murakami Y, Baba T, et al. Bactericidal/Permeability-Increasing Protein Promotes Complement Activation for Neutrophil-Mediated Phagocytosis on Bacterial Surface. Immunology (2001) 103(4):519-25. doi: 10.1046/j.1365-2567.2001.01263.x
36. Heale JP, Pollard AJ, Stokes RW, Simpson D, Tsang A, Massing B, et al. Two Distinct Receptors Mediate Nonopsonic Phagocytosis of Different Strains of Pseudomonas aeruginosa. J Infect Dis (2001) 183(8):1214-20. doi: 10.1086/ 319685

37. Wilson ZS, Ahn LB, Serratelli WS, Belley MD, Lomas-Neira J, Sen M, et al. Activated $\beta 2$ Integrins Restrict Neutrophil Recruitment During Murine Acute Pseudomonal Pneumonia. Am J Respir Cell Mol Biol (2017) May56 (5):620-7. doi: 10.1165/rcmb.2016-0215OC

38. Bednarczyk M, Stege H, Grabbe S, Bros M. $\beta 2$ Integrins-Multi-Functional Leukocyte Receptors in Health and Disease. Int J Mol Sci (2020) 21(4):1402. doi: 10.3390/ijms21041402

39. Alexis NE, Muhlebach MS, Peden DB, Noah TL. Attenuation of Host Defense Function of Lung Phagocytes in Young Cystic Fibrosis Patients. J Cyst Fibros (2006) 5(1):17-25. doi: 10.1016/j.jcf.2005.11.001

40. Simonin-Le Jeune K, Le Jeune A, Jouneau S, Belleguic C, Roux PF, Jaguin M, et al. Impaired Functions of Macrophage From Cystic Fibrosis Patients: CD11b, TLR-5 Decrease and sCD14, Inflammatory Cytokines Increase. PLoS One (2013) 8(9):e75667. doi: 10.1371/journal.pone.0075667

41. Chalmers JD, Ma A, Turnbull K, Doherty C, Govan JR, Hill A. Impaired Neutrophil Phagocytosis and Receptor Expression in non-CF Bronchiectasis. Eur Respir J (2013) 42:P2065.

42. Hodge S, Hodge G, Scicchitano R, Reynolds PN, Holmes M. Alveolar Macrophages From Subjects With Chronic Obstructive Pulmonary Disease are Deficient in Their Ability to Phagocytose Apoptotic Airway Epithelial Cells. Immunol Cell Biol (2003) 81:289-96. doi: 10.1046/j.1440-1711.2003.t011-01170.x

43. Berenson CS, Garlipp MA, Grove LJ, Maloney J, Sethi S. Impaired Phagocytosis of Nontypeable Haemophilus Influenzae by Human Alveolar Macrophages in Chronic Obstructive Pulmonary Disease. J Infect Dis (2006) 194:1375-84. doi: 10.1086/508428

44. Garcia-Vidal C, Almagro P, Romaní V, Rodríguez-Carballeira M, Cuchi E, Canales L, et al. Pseudomonas aeruginosa in patients hospitalised for COPD exacerbation: a prospective study. Eur Respir J (2009) 34(5):1072-8. doi: $10.1183 / 09031936.00003309$

45. Taylor AE, Finney-Hayward TK, Quint JK, Thomas CM, Tudhope SJ, Wedzicha JA. Defective Macrophage Phagocytosis of Bacteria in COPD. Eur Respir J (2010) 35:1039-47. doi: 10.1183/09031936.00036709

46. Balakrishnan A, Schnare M, Chakravortty D. Of Men Not Mice: Bactericidal/ Permeability-Increasing Protein Expressed in Human Macrophages Acts as a Phagocytic Receptor and Modulates Entry and Replication of Gram-Negative Bacteria. Front Immunol (2016) 7:455. doi: 10.3389/fimmu.2016.00455

47. Tian Y, Zeng T, Tan L, Wu Y, Yu J, Huang J, et al. Clinical Significance of BPIANCA Detecting in COPD Patients With Pseudomonas aeruginosa colonization. J Clin Lab Anal (2019) 33(6):e22908. doi: 10.1002/jcla.22908

48. Theprungsirikul J, Thaden JT, Wierzbicki RM, Burns AS, Skopelja-Gardner S, Fowler VGJr, et al. Low-Avidity Autoantibodies against Bactericidal/ Permeability-Increasing Protein Occur in Gram-Negative and GramPositive Bacteremia. Infect Immun (2020) 88(10):e00444-20. doi: 10.1128/ IAI.00444-20

49. Rosales C. Neutrophil: A Cell with Many Roles in Inflammation or Several Cell Types? Front Physiol (2018) 9:113. doi: 10.3389/fphys.2018.00113

50. Jasper AE, McIver WJ, Sapey E, Walton GM. Understanding the Role of Neutrophils in Chronic Inflammatory Airway Disease. F1000Res (2019) 8(F1000 Faculty Rev):557. doi: 10.12688/f1000research.18411.1

Conflict of Interest: The authors declare that the research was conducted in the absence of any commercial or financial relationships that could be construed as a potential conflict of interest.

Copyright (c) 2021 Theprungsirikul, Skopelja-Gardner, Burns, Wierzbicki and Rigby. This is an open-access article distributed under the terms of the Creative Commons Attribution License (CC BY). The use, distribution or reproduction in other forums is permitted, provided the original author(s) and the copyright owner(s) are credited and that the original publication in this journal is cited, in accordance with accepted academic practice. No use, distribution or reproduction is permitted which does not comply with these terms. 